\title{
Reclamos de los consumidores y calidad de los productos: análisis de estática comparativa.
}

\author{
Joaquín Coleff*
}

\begin{abstract}
Existe una variada evidencia empírica que documenta que muchos consumidores no reclaman compensación o reemplazo cuando descubren que el producto adquirido presenta una falla. Este hecho reduce los costos de las empresas que pueden tener incentivos a bajar la calidad de los productos. Desde los sesenta las asociaciones de consumidores comenzaron a fomentar a que los consumidores ejerzan sus derechos. En este trabajo analizamos las implicancias de reducir el costo de reclamar de los consumidores en las deciciones sobre precio y calidad de empresas monolísticas. Encontramos que la empresa puede elegir producir un producto de menor calidad cuando estas asociaciones están presentes. El mecanismo es el siguiente: la reducción en los costos de reclamar no solo incrementa la proporción de reclamantes entre los compradores sino que también incrementa el deseo a pagar por el producto. Este segundo efecto surge porque es más barato garantizarse un producto bueno a través de los reclamos, el cuál generate que menor sensibilidad de la demanda a la calidad del producto. Un incremento en el número de reclamos motiva incrementar la calidad, pero la reducción en la sensiblidad de la demanda motiva reducir la calidad. Cuando el segundo efecto domina la calidad cae. Proveemos evidencia sobre los Recalls en el mercado de autos de U.S.A. con concilia nuestros resultados.
\end{abstract}

Clasificación JEL: K42,D71, D42, D21, L12.

\section{Introduction}

Existe una variada evidencia empírica (Véase Best and Andreasen (1977) y Huppertz (2007) entre otros) que documenta que muchos consumidores no reclaman compensación o reemplazo cuando descubren que el producto adquirido presenta una falla. Huppertz (2007), basándose en Hirschmann (1970), racionaliza este comportamiento aduciendo que los consumidores tienen un alto costo de reclamar. $\mathrm{Si}$ este es el caso, cualquier mecanismo que reduzca el costo de reclamar, como el actuar de las asociciaciones de consumidores, debería incrementar el número de reclamos recibidos por las empresas, incrementando sus costos marginales (producción más atención post-venta) y debería motivar una mejora en la calidad de los productos ofrecidos por las empresas. En este trabajo, sin embargo, probamos que el efecto puede ser el opuesto: la fracción de productos con fallas manufacturados puede aumentar.

*Director: Walter Cont 
En los 60s, las asociaciones de consumidores (en adelante ACs) se hicieron populares. ${ }^{1}$ Desde entonces, ACs han estado actuando entre los consumidores, las empresas y los políticos: han logrado aprovar leyes que agregan derechos de los consumidores, han suministrado información fiable sobre derechos, procedimientos, etc. a los consumidores, y han representado los intereses de los consumidores en acciones directas. Algunas agencias del gobierno, como la National Highway Traffic Safety Administration (NHTSA) en USA, también proveen un servicio a los consumidores: esta agencia recoleta información acerca de productos (autos, camiones, motos, etc.) y fuerza a que las compañias hagan un "recall" (llamado a los consumidores), e.g. reparar, reemplazar o re-comprar productos con fallas. Los consumidores pueden considerar este procedimiento como sustituto o complementario de las costosas acciones legal directas.

Aunque estas organizaciones han alcanzado popularidad, no existe evidencia empírica o análisis teórico del que esté al tanto que identifique los efectos de las asociaciones de consumidores en la performance del mercado. Algunas excepciones son Inderst and Ottaviani (2009) mostrando que la imposición de condiciones mínimas en políticas de devolución de las empresas puede mejorar el bienestar y el excedente de los consumidores cuando la proporción de compradores crédulos es alta; y Xinyu (Forthcoming JLEO) mostrando que cuando la responsabilidad de las firmas está sujeta a la acción de un recall existe mayor respuesta de los consumidores a los recalls (a asistir a la reparación, reemplazo o recompra) pero que las empresas tienen menores incentivos a realizar los recalls de forma voluntaria.

El efecto de reducir el costo de reclamar de los consumidores en el número de quejas puede ser muy importante. Por ejemplo, en la Figura 1.a vemos la evolución de las quejas de consumidores recibidos por la National Highway Traffic Safety Administration (NHTSA) para el mercado de autos de U.S.A. ${ }^{2}$ (línea verde). La NHTSA es la agencia del gobierno de U.S.A. encargada de reglamentar y controlar los estándares de seguridad vial en el mercado de autos. En 1995, esta agencia ha incorporado el correo eléctronico y un dispositivo on-line como dos mecanismos baratos para que los consumidores realicen reclamos. Desde entonces el número de quejas se ha incrementado considerablemente. Además, el número de reclamos se relaciona positivamente con el número de recalls ${ }^{3}$ (línea negra).

Para estudiar el efecto de la reducción en el costo de reclamar generado por la ACs, construimos un modelo donde una empresa monopolista elige precio y calidad. La calidad se define como la probabilidad que el producto tenga un fallo. Atender un reclamo y proveer mejor calidad son acciones costosas para la empresa. Los consumidores tiene una utilidad alta cuando el producto tiene la performance esperada y una utilidad baja cuando el producto tiene una falla. Los consumidores difieren en la utilidad del producto sin falla. Si el producto es defectuoso, ellos deciden si reclamar una compensación incurriendo en un costo de reclamo. Para simplificar el modelo, suponemos que todos los consumidores tienen el mismo costo de reclamar. En este contexto, proponemos un análisis de estática comparativa para comprender el

\footnotetext{
${ }^{1}$ Las primeras organizaciones que se centraron en los derechos de los consumidores fueron The National Consumer League fundada en 1899 y la Consumer Union fundada en 1936. Sin embargo no fue hasta los 60s que las asociaciones se hicieron populares y conocidas en varios de los países desarrollados y en vías de desarrollo.

${ }^{2}$ La información corresponde a las 6 compañías más grandes: Ford, General Motors, Chrysler, Toyota, Nissan and Honda. Estas compañias tienen el $90 \%$ del mercado de autos de U.SA. desde 1970. La NHTSA provee la información de las quejas desde 1984.

${ }^{3}$ En otro contexto, Owens and Hausknecht (1999) realizan un experimento con la Council of Better Business Bureau en U.S.A. y muestran que simplificando el proceso para reclamar aumenta considerablemente la tasa de reclamos. Ellos encuentran que la tasa de respuesta aumenta de $22 \%$ a $48 \%$ cuando introducen un procedimiento más simple. Además, la satisfacción de los consumidores con el procedimiento más simple aumenta aún cuando no estén satisfechos con el resultado del reclamo.
} 


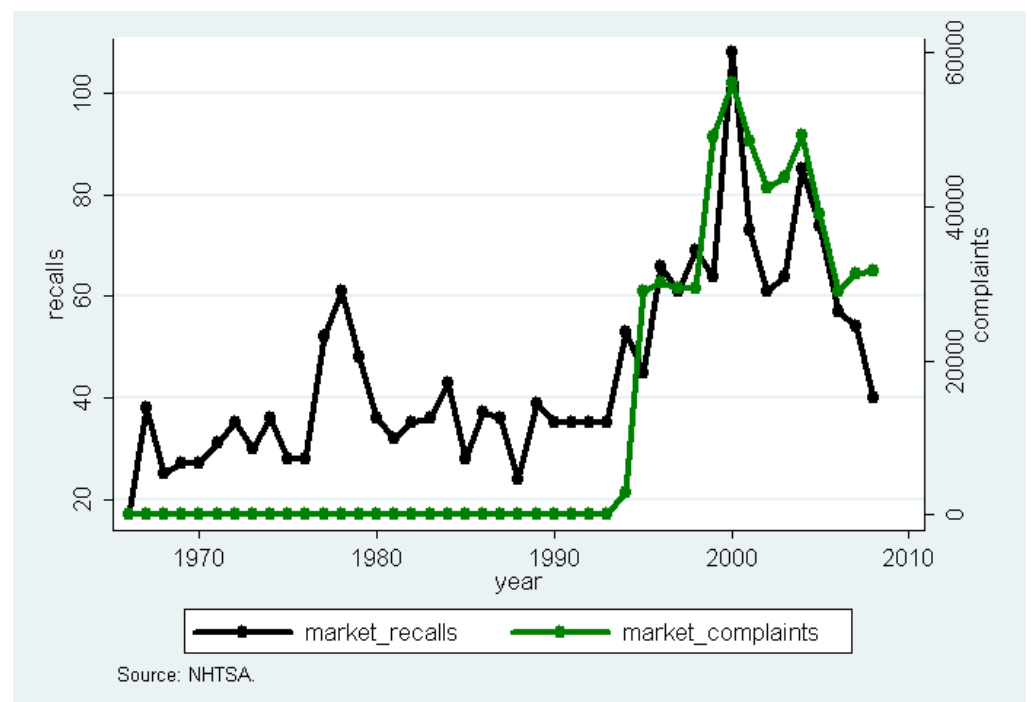

Figure 1: Número de "Recalls" y reclamos por año para el mercado de autos de U.S.A.

impacto de las ACs de reducir el costo de reclamar en la performance del mercado. ${ }^{4}$

Por el lado de la demanda, los consumidores son reclamantes si solicitan compensación cuando reciben productos defectuosos y no reclamantes en caso contrario. Los consumidores con mayor deseo a pagar tienen mayor probabilidad de ser reclamantes. Un incremento en el precio no solo reduce el número de compradoers sino que también incrementa la proporción de reclamantes entre los compradores. Entonces, existe una relación directa entre la proporción de reclamantes entre compradores y el precio. Por el lado de la oferta, el costo marginal esperado de la empresa, producción más atención post-venta, depende de la proporción de reclamantes entre compradores. La firm tiene un costo postventa si hay un reclamo, i.e. si un reclamante compra un producto que resulta ser defectuoso. En consecuencia, el costo esperado de la empresa es endógeno y está determinado por la calidad y el precio.

Dado el precio y la calidad, la proporción de reclamantes y no reclamantes entre compradores está determinada por el costo de reclamar. Entonces, la estrategia de precios depende del costo de reclamar de los consumidores: cuando el costo de reclamar es alto, hay muchos no reclamantes y la empresa elige una estrategia de precio bajo para atraer más compradores que nunca realizar quejas. Cuando el costo de reclamar es bajo, hay muchos reclamantes y la empresa elige una estrategia de precio alto. Hay un relación monótona inversa entre la estrategia de precios óptima y el costo de reclamar.

En la decisión de calidad, la empresa enfreta un tradeoff con tres efectos distintos. Por un lado, aumentar la tasa de productos con fallos reduce el costo marginal de producción. Pero también aumenta el número de quejas que la empresa recibirá y por ende el número de compensaciones que tendrá que efectuar (efecto reclamos). Por otro lado, aumentar la tasa de fallos reduce el deseo a pagar de los consumidores por el producto y, consecuentemente, reduce la demand del producto (efecto demanda).

\footnotetext{
${ }^{4}$ Como todos los consumidores tienen el mismo costo de reclamar no podemos distinguir el efecto de reducir el costo de reclamar debido a que las asociaciones de consumidores proveen información gratuita, o debido una asistencia directa a bajo costo de las asociaciones de consumidores (pagando una tarifa periódica). Sin pérdida de generalidad, todos los consumidores eligen entre unirse a la AC o reclamar de forma privada cuando reciben un producto defectuoso utilizando la información gratuita.
} 
Las ponderaciones de cada efecto (reclamos y demanda) depende de la estrategia de precios elegida por la empresa: para una estrategia de precio alto hay un efecto reclamo fuerte y un efecto demand débil que aumenta con el costo de reclamar. Sin embargo, para una estrategia de precio bajo hay un efecto demanda fuerte y un efecto reclamo débil que decrece con el costo de reclamar. La estrategia de calidad óptima es creciente en el costo de reclamar hasta un valor crítico por encima del cuaal es decreciente en el costo de reclamar (forma de U).

Un equilibrio consistente con la evidencia empírica donde una proporción de los consumidores nunca reclama se puede representar en nuestro modelo por una situación con alto costo de reclamar donde la firma elige una estrategia de precio bajo. Suponga que gracias a las asociaciones de consumidores se genera una reducción fuerte en el costo de reclamar. Si este es el caso, la empresa aumentará el precio reduciendo el número de compradores y aumentando la proporción de reclamantes entre compradores. En la estrategia de calidad, la reducción del efecto demanda puede compensar el incremento del efecto reemplazo y la calidad empeorar.

An equilibrium consistent with empirical evidence where some proportion of buyers never request a replacement is represented in our model by a situation with high consumers' claiming cost followed by a low price strategy for the firm. Now, suppose that a consumers' association exists generating a nonlocally reduction in consumers' claiming cost. If this is the case, the firm increases the price reducing the number of buyers and increasing the proportion of claimants among buyers. In the reliability strategy, the reduction in the demand effect offsets the increment in the replacement effect and reliability decreases.

Lo que origina este resultado es la heterogeneidad en los consumidors y un bajo costo de atención post-venta. Sin la heterogeneidad en los consumidores no existe diferencia entre reclamantes y no reclamantes entre compradores, y consecuentemente no hay cambio en la estrategia de precio de la empresa. Si este fuera el caso la calidad del producto guarda una relacióno monotónica con el costo de reclamar de los consumidores. Por otro lado, si el costo de recibir reclamos de la empresa es extremadamente alto la reducción en el efecto demanda nunca compensará el incremento en el efecto reclamo. En este caso, la calidad del producto aumentará con la intervención de las ACs.

En el mercado automotriz de U.S.A, la NHTSA es la agencia gubernamental que regula y controla los estándares de seguridad vial. Luego de la introducción de dispositivos de quejas on-line, hubo un aumento en el número de reclamos y el número de "Recalls" realizados por las empresas. Los consumidores usan el servicio de la NHTSA como un mecanismo adicional para expresar su disconformidad. Si las compañías no atienden sus reclamos los consumidores eligen entre: realizar acciones legales contra la empresa, contactar una associación de consumidores (como la Consumer Unioni), reclamar por medio de la NHTSA, una combinación de las anteriores o no hacer nada. Además, aún cuando reciban un compensación, algunos consumidores pueder presentar quejas a las NHTSA en solidaridad con el resto de los consumidores.

La Figura 1 muestra la evolución de las quejas y los recalls por año en el período 1970-2008. Las aplicaciones on-line de la NHTSA ayudó a reclamar a los compradores de autos producidos tanto antes como después de 1995. Sin embargo, como luego se prueba, el número de quejas y recalls se incrementó en mayor medida para los autos producidos luego de 1995. Este efecto es consistente con nuestras predicciones que la reducción en el costo de reclamar de los consumidores ha motivado a las firmas a reducir la calidad de los productos manufacturados.

Este trabajo se relaciona con varias líneas de la literatura. La literatura en responsabilidad legal de los 
productos es extensa. Murthy and Djamaludin (2002) and Huang, Liu, and Murthy (2007) analizan como las garantías pueden afectar la decisión de las empresas en la calidad y en el número de reclamos hechos por los consumidores. Oi (1973) analiza como un cambio en la responsabilidad legal del consumidor al vendedor puede afectar la calidad negativamente. Daughety and Reinganum (2008) trabajanen la relación entre el precio como señal y la revelación de información cuando la seguridad del producto es información privada. Daughety and Reinganum (1995) estudian la relación entre la fase de I\&D y la seguridad del producto cuando el precio puede funcionar como señal de la seguridad del producto. Daughety and Reinganum (2005) analizan la relación entre seguridad, I\&D and arreglos confidencial en los juicios. Nuestra contribución se centra en mostrar que existe un relación endógena entre la proporción de reclamantes entre los compradores y el precio del producto. Esta relación se translada a los costos marginales de la empresa y a la decisión óptima de calidad.

La literatura sobre el comportamiento de reclamos de los consumidores (consumer complain behavior (CCB) en inglés) se focaliza en la reacción de los consumidores ante al insatisfacción. (Chebat, Davidow, and Codjovi (2005)). En este trabajo nos concentramos en los reclamos realizados ante las empresas o terceras partes (ACs) asumiendo que no existe otra forma de expresar la insatisfacción que pueda afectar a la empresa. Asumimos que la empresa asume un costo adicional por reclamo y que la información es pública. Nuestra contribución a esta literatura es mostrar que el número de reclamos a terceras partes esta determinado endógenamente y que la calidad del producto puede tanto mejorar como empeorar cuando se reduce el costo de reclamar de los consumidores.

Algunos mecanismo alternativos que reducen el costo de reclamar de los consumidores son las acciones legales colectivas (Class Action Lawsuit, véase Hensler (2001) y Klement and Neeman (2004)) y las cortes pequeñas (Small Courts, véase Best and Andreasen (1977)). Klein and Leffler (1981) estudian la elección de fiabilidad en un contexto dinámico. Cuando las compras son repetidas la reputación y las marcas pueden garantizar la provisión de producto de alta calidad. Finalmente, Greif, Milgrom, and Weingast (1994) describen como las redes de comerciantes pueden mejorar los términos de comercio (como la calidad o fiabilidad del producto).

\section{Modelo}

Consideremos un monopolista que maximiza sus beneficios eligiendo precio y calidad. Los consumidores eligen si comprar una unidad del producto y si reclamar para que reemplacen el producto en caso de que este esté fallado. Los consumidores son heterogéneos en la valuación del producto si este funciona normalmente y homogéneos en el costo de solicitar un reemplazo/compensación. Este costo de reemplazo captura, entre otros, el costo de oportunidad del tiempo. Assumimos que el reemplazo está garantizado.

La secuencia es la siguiente: la empresa elige el precio, $p$, y la calidad, $x$. Cada consumidor observa la elección de la empresa y decide si comprar el producto o no. ${ }^{5}$ Luego de comprar, los consumidores observan si el producto está fallado. La probabilidad de que el producto esté fallado es $x$. Finalmente, los consumidores solicitan un reemplazo, que está garantizado, o no.

\footnotetext{
${ }^{5} \mathrm{El}$ supuesto de que la calidad es revelada sin costo y que los consumidores creen que si no se revela, la calidad es la peor posible. Esto permite concentrarme en el punto principal de este trabajo.
} 
El modelo es similiar al considerado por Bar-Isaac, Caruana, and Cuñat (2009). En su trabajo, ellos se concentran en la adquisición de información sobre cuán adecuado es el producto a través de estrategias de marketing antes de realizar la compra. Aquí el producto funciona (es bueno) o está fallado (es malo) y los compradores eligen, luego de comprar, si reclamar un reemplazo o no.

\subsection{Demanda}

Tenemos un continuo de consumidores heterogéneous de masa 1. Cada consumidor $i$ está caracterizado por una dupla $\left(k, v_{i}\right)$, donde $k \in[0,1]$ es el costo de reclamar compensación si el producto falla y $v_{i}$ es la valuación del consumidor $i$ de un producto que funciona correctamente. Si el producto está fallado el consumidor tiene una utilidad de 0 . Supongamos que $v_{i}$ está distribuido uniformemente en $[0,1]$. La utilidad de reserva de todos los consumidores es 0 .

Cada consumidor anticipa que, cada vez que reciba un producto defectuoso, reclamrá una compensación si $v_{i}-k \geq 0$. Entonce, dado $p$, y $x$, un consumidor qeu compra una unidad del producto recibe una utilidad de $v_{i}-p$ con probabilidad $(1-x)$ y una utilidad de $-p$ con probabilidad $x$. Sin embargo, si el producto falla, el consumidor tiene una utilidad adicional de $v_{i}-k$ si $v_{i}>k$. Entonces, la experanza de la utilidad del consumidor $i$ por comprar el producto es

$$
E U_{i}(p, x)=(1-x) v_{i}-p+x \max \left\{v_{i}-k, 0\right\}
$$

Comprar es la decisión óptima si $E U_{i}(p, x) \geq 0$. Dado $p$ y $x$, podemos encontrar la función de demanda, $Q(p, x)$, y la función de reemplazos, $\hat{Q}(p, x)$, que describe el número de consumidores que replazarán el producto si el que comprar está fallado. Las Figuras Figures 2.a y 2.b. representan las elecciones de los consumidores.

Aquellos consumidores con $v_{i} \geq k$ anticipan que reemplazarán el producto si lo compran y resulta estar fallado. De estos consumidores, los que satisfacen $v_{i} \geq p+x k$ compran el producto. Los consumidores con $v_{i}<k$ anticipan que no reemplazarán el producto. De estos, aquellos con $v_{i} \geq \frac{p}{1-x}$ compran el producto.

El valor de $p$ puede ser tal que $p \gtrless k(1-x) . k(1-x)$ es el punto donde las funciones de demand y reemplazos tienen un quiebre. Cuando $p>k(1-x)$, como $p_{H}$ en las Figuras 2.a y 2.b, todos los compradores reclaman una compensación siempre que el producto esté fallado. Cuando $p<k(1-x)$, como $p_{L}$ en las Figuras 2.a y 2.b, algunos consumidores reclaman y otros no reclaman conpensación si el producto está fallado.

Agregando en las decisiones de todos consumidores construimos $Q(p, x)$ y $\hat{Q}(p, x)$ que son,

$$
Q(p, x)= \begin{cases}0 & \text { si } p \geq 1, \\ \max \{1-p-x k, 0\} & \text { si } k(1-x) \leq p<1, \\ \max \left\{1-\frac{p}{1-x}, 0\right\} & \text { si } 0<p<k(1-x)<1, \\ 1 & \text { si } p \leq 0\end{cases}
$$




$$
\hat{Q}(p, x)= \begin{cases}0 & \text { si } p \geq 1, \\ \max \{1-p-x k, 0\} & \text { si } k(1-x)<p<1, \\ \max \{1-k, 0\} & \text { si } p<k(1-x) \leq 1\end{cases}
$$

$x \hat{Q}(p, x)$ representa la suma esperada de todas las reclamaciones recibidas por la empresa: cada consumidor que reclama una compensación recibirá un producto fallado con probabilidad $x$.

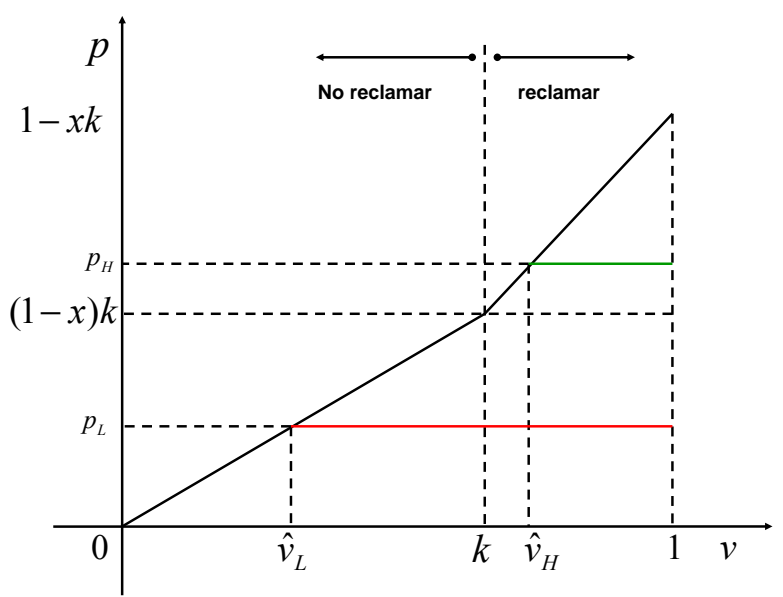

(a) Maximo deseo a pagar para cada $v_{i}$.

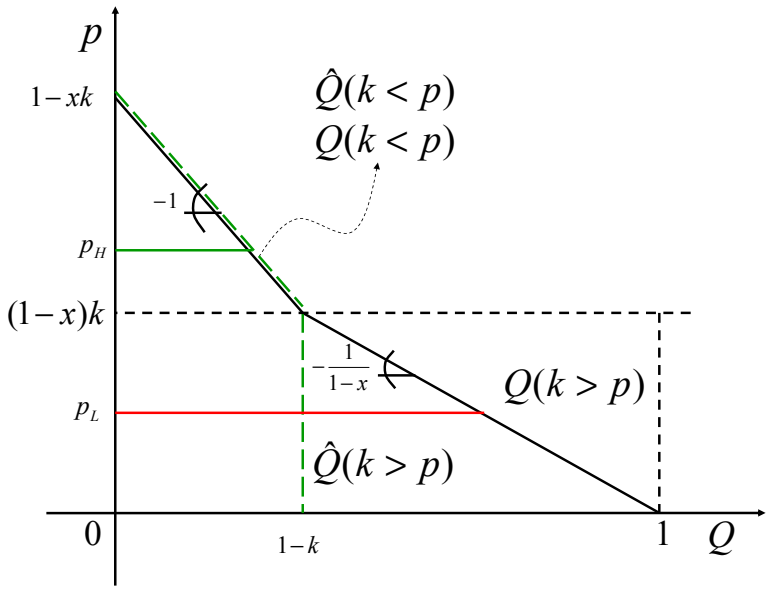

(b) Funciones de Demanda (negro) y Reclamos (verde)

Figure 2: Decisión óptima de los consumidores dado $p$ y $x$

Note que el costo de reclamar de los consumidores determina que consumidores serán reclamantes y que consumidores serán no reclamantes si compran el producto. En otras palabras, el costo de reclamar determina no solo el quiebre en la demanda sino también el effecto que precio tiene en la proporción de reclamantes entre los compradores. Un producto con precio elevado es comprado solo por aquellos consumidores que valoran mucho el producto. Estos comprados son muy probablemente los que encuentren beneficiosos reclamar una compensación si el producto falla. Un producto con precio bajo es comprado por consumidores con alta y baja valoración por el producto. Probablemente, los consumidores con baja valoración no soliciten compensación si el producto falla. Entonces un precio más bajo reduce la proporción de reclamantes sobre los compradores. Por otro lado, si la calidad del producto aumenta la demanda se desplaza hacia arriba.

\subsection{Problema del Monopolista}

El monopolista maximiza beneficios eligiendo $p$ y $x$. El costo marginal de producción es $c(x)$, con $c^{\prime}(x)<0$ y $c^{\prime \prime}(x)>0$, y el costo de compensación por unidad es $c_{r} \geq 0$ que está determinado por el sistema de derecho. Dadas las funciones de demand y de reemplazos en las ecuaciones (2) y (3), podemos escribir el costo promedio agregado $c(x)+x c_{r} \frac{\hat{Q}}{Q}$.

Dadas las funciones de demanda y reemplazos el problema de maximización del monopolista es ${ }^{6}$

\footnotetext{
${ }^{6}$ En otra versión de este trabajo utilizamos otras formas para modelar reclamos. El reclamo genera el reemplazo del producto por uno nuevo. En una versión, solo se permite un reemplazo, pero en otra versión el comsumidor puede realizar tantos reemplazos como sea necessario hasta recibir un producto que no esté fallado. En el caso de un reemplazo el costo por unidad
} 


$$
\max _{p, x}\left[p-\left(c(x)+x c_{r} \frac{\hat{Q}(p, x)}{Q(p, x)}\right)\right] Q(p, x) \quad \text { sujeto a } \quad(p, x) \in \mathbb{R}_{+} \times[0,1] .
$$

Como los consumidores valoran el producto por lo menos $0 \mathrm{y}$ a lo sumo 1 , el precio de un equilibrio no trivial siempre satisface $p \in(0,1) .{ }^{7}$ El costo marginal agregado de la firma, costo de producción y de transacción, no es constante. Dado $x$, el costo marginal agregado es no decreciente en $p$. En este caso es un función escalonada que tiene el valor de $c(x)+x c_{r}$ para $p>k(1-x)$ y tiene el valor de $c(x)$ para $p<k(1-x)$. Esto se explica porque hay un ordanamiento entre reclamantes y no reclamante. Los consumidores que siempre reclaman compensación si el producto está fallado son los que tienen mayor deseo a pagar. El consumidor marginal es un reclamante si $p>k(1-x)$. Los que nunca reclaman el producto tienen menor deseo a pagar. El consumidor marginal es no reclamante si $p<k(1-x){ }^{8}$

El costo medio es $c(x)+x c_{r} \underline{Q}$. Es creciente en $p$ cuando $p<k(1-x)$. La firma vende a un grupo de compradores donde una proporción $\frac{\hat{Q}}{Q}$ son reclamantes. La empresa obtiene un margen unitario alto de los no reclamantes, i.e. $p-c(x)$ y un margen unitario menor de los reclamantes,i.e. $p-c(x)-x c_{r}$, con una proporción $\frac{\hat{Q}}{Q}$ de reclamantes. ${ }^{9}$ Dado este efecto adicional en el precio, la decisión del precio pondera el tradicional trade off: costo marginal versusingreso marginal.

En la decisión de calidad hay 3 fuerzas diferentes. Por un lado, incrementar tasa de fallos reduce el costo marginal de producción. Sin embargo, incrementa el número de veces que la firma recibe reclamos y, por ende, el número de compensaciones que la firma efectuará (efecto reclamos). Por otro lado, incrementar la tasa de fallos reduce la valuación de los consumidores por el producto y, por ende, reduce la demand del producto (efecto demanda): si el consumidor anticipa que reclamará tendrá seguro una valuación alta del producto pero eventualmente tendrá que realizar una reclamación. En cambio, si el consumidor anticipa que no reclamará, el valuación esperada será menor y consecuentemente tendrá menor deseo a pagar. La ecuación (5) refleja estos tradeoffs en la decisión de calidad,

$$
-c^{\prime}(x) Q=c_{r} \hat{Q}-\frac{\partial Q}{\partial x}\left[p-c(x)-x c_{r} \frac{\frac{\partial \hat{Q}}{\partial x}}{\frac{\partial}{\partial x}}\right] .
$$

Los ponderadores de cada efecto (efecto reclamo y demanda) dependen de la estrategia de precio que elija al empresa: para una estrategia de precio alto hay un efecto reclamo fuerte y un efecto demand débil que incrementa con el costo de reclamar de los consumidores. Una estrategia de precio bajo tiene un efecto demand fuerte y un efecto reclamo débil decreciente en el costo de reclamar de los consumidores.

es $x\left(c(x)+c_{r}\right)$, mientras que en el caso de muchos reemplazos el costo es $\frac{x\left(c(x)+c_{r}\right)}{1-x}$. En todas las versiones los resultados se mantienen.

${ }^{7}$ La función de beneficios $\Pi:[0,1] \times[0,1] \rightarrow \mathbb{R}$ es continua y definida en un subconjunto compacto de $\mathbb{R}^{2}$. Por el teorema de Brauwer, $\Pi$ tiene un máximo global en $[0,1] \times[0,1]$.

${ }^{8}$ En caso de heterogenidad en el costo de reclamar compensación habrá ordanamiento estadístico: consumidores con mayor deseo a pagar tienen más propensión a reclamar que consumidores con menor deseo a pagar.

${ }^{9} \mathrm{El}$ ordenamiento de los consumidores refleja el supuesto que los consumidores que tiene mayor deseo a pagar son los que más pierden cuando reciben un producto fallado, y por ende los que reclaman. Los resultados principales del modelo depende de este supuesto. Sin embargo, podemos introducir heterogeneidad en el costo de reclamar de los consumidores para obtener mayor diversidad en las decisiones de compra y reclamo, sin que se alteren los principales resultados. 
La decisión óptima de calidad presenta un forma en $\mathrm{U}$ en el costo de reclamar de los consumidores.

La empresa maximiza el beneficio siguiendo una de las posible estrategias dependiendo del parámetro $k$ : a) $p>k(1-x)$ o b) $p<k(1-x)$. El primer tipo de estrategia está caracterizada por un precio alto (baja demanda) que garantiza a alto margen por unidad vendida. Solo los consumidores con alto deseo a pagar compran el producto y siempre reclaman compensación cuando el producto falla. El segundo tipo de estrategia está caracterizada por un precio bajo (alta demanda) que permite a muchos consumidores comprar el producto aunque algunos no reclamen compensación si el producto falla. En este caso hay dos tipos de compradores, los que compran y siempre reclaman si el producto falla (bajo margen debido a alto costo marginal) y los que compran y nunca reclaman (alto margen debido a bajo costo marginal).

El valor de $k$ determina qué estrategia seguirá la firma para maximizar sus beneficios. La estrategia de precio alto es óptima para valores de $k$ bajos y la estrategia de precio bajo es óptima para valores de $k$ altos. A mayor $k$ habrá mayor proporción de consumidores que nunca reclaman compensación cuando el producto está defectuoso.

En la siguiente sección explicamos detalladamente las estrategias para comprender la decisión óptima de la empresa. Sin embargo, algunos supuestos técnicos son necesarios sobre $c(x)$ para que el problema esté bien definido. El valor de $x_{0}$ es el que satisface la siguiente condición $-c^{\prime}\left(x_{0}\right)=\frac{1-x_{0}+c\left(x_{0}\right)}{2\left(1-x_{0}\right)} \cdot{ }^{10}$

$$
\text { A0 - } 1<c(x=0) \text {. }
$$

$\mathrm{A} 1-1>c\left(x_{0}\right)+x_{0}$.

El supuesto A0 implica que el costo de producción es muy elevado cuando $x=0$, entonces el único equilibrio posible es trivial con un precio suficientemente elevado tal que no hay mercado $(Q=0)$. A1 dice que un incremento en la tasa de fallos reduce el costo de producción en al menos un intervalo. En este intervalo la reducción en costos es mayor que la reducción en el deseo a pagar de algunos consumidores. A1 garantiza que si ningún consumidor realiza reclamos es beneficioso para la firma producir y vender el producto.

\subsection{Estrategias de la empresa}

La empresa puede elegir una estrategia de precio alto o bajo. Utilizamos el subíndice $L$ para precio Bajo, $\left(p_{L}, x_{L}\right)$, y subíndice $H$ para precio alto, $\left(p_{H}, x_{H}\right)$. En el Lema 1 concluimos que si la función de beneficios no es diferenciable en $[0,1]$ no existe equilibrio con una solución de esquina en $p$.

Lemma 1. Dados los supuestos A0-A1, si la función de beneficio no es diferenciable, no existe equilibrio con soluciones de esquina. ${ }^{11}$ i.e. no existe equilibrio donde $p=0, p=k(1-x)$ or $p=1$.

Gracias al Lema 1 buscamos el equilibrio interior donde $(1-x) k<p<1$ o $0<p<(1-x) k$. Asociamos la estrategia de precio alto con $(1-x) k<p<1$ y al estrategia de precio bajo con $0<p<(1-x) k$. Desarrollamos ambas estrategias y luego determinamos cuál es la que maximiza los beneficios de la empresa. En las condiciones de primer order respecto de $p$ encontramos el precio óptimo como función de

\footnotetext{
${ }^{10}$ Este valor es la tasa de fallos óptima cuando ningún consumidor realiza reclamos (o $\left.k \rightarrow+\infty\right)$.

${ }^{11}$ Note que existe un valor de los parametros para el cuál la función de beneficios es diferenciable para todo $k \in(0,1)$. En este caso, las condiciones de primer orden caracterizan el equilibrio no trivial.
} 
$x, p(x)$, y luego lo reemplazamos en la función de beneficio. Suponemos que la solución existe y luego verificamos que la solución satisface las restricciones. Este procedimiento se debe a ventajas algebraicas.

\subsubsection{Estrategia de precio alto}

Hay 3 condiciones necesarias para que la empresa elija una estrategia de precio alto: $\mathrm{C} 1--c^{\prime}(x)=c_{r}+k$ para el $x$ óptimo; C2- $1-c(x)-x c_{r}-x k \geq 0$ para beneficios no negativos; y C3- $k \leq k_{H} \equiv \frac{1+c(x)+x c_{r}}{2-x}$ para satisfacer la restricción, i.e. $p>(1-x) k$. La condición $\mathrm{C} 1$ implica que la solución óptima $x_{H}$ satisface $\frac{\partial x_{H}}{\partial k}<0$ y $\frac{\partial x_{H}}{\partial c_{r}}<0$ monotonicamente. Si la empresa elige una estrategia de precio alto los beneficios son

$$
\Pi=\left(\frac{1-c\left(x_{H}\right)-x_{H} c_{r}-x_{H} k}{2}\right)^{2} \quad \text { si } k \leq k_{H} \equiv \frac{1+c\left(x_{H}\right)+x_{H} c_{r}}{2-x_{H}} .
$$

Por el teorema de la envolvente, $\frac{\partial \Pi}{\partial k}<0$ y $\frac{\partial \Pi}{\partial c_{r}}<0$ monotonicamente. Dado $c_{r}$ hay una cota superior $\underline{k}_{H}\left(c_{r}\right)$ tal que para todo $k \leq \underline{k}_{H}\left(c_{r}\right)$ la estrategia de precio alto satisface 6. También existe una cota superior en $c_{r}$ para que la firma obtenga beneficios no negativos. Para encontrar la cota superior $\underline{k}_{H}\left(c_{r}\right)$ analizamos los distintos casos posibles.

Si las condiciones $\mathrm{C} 1$ y C2 se satisfacen en igualdad, podemos encontrar $-c^{\prime}\left(x_{H 1}\right)=\frac{1-c\left(x_{H 1}\right)}{x_{H 1}} . x_{H 1}$ es el valor de $x$ elegido por la empresa para maximizar sus beneficios que son iguales a 0 (por $\mathrm{C} 2$ ). Reemplazando $x_{H 1}$ en la condición C2 encontramos que $k_{H 1}=-c^{\prime}\left(x_{H 1}\right)-c_{r}$. Dado $c_{r}, k_{H 1}$ es el máximo valor de $k$ tal qe la firma alcanza beneficios no negativos. Si, adicionalmente, la condición C3 se satisface en igualdad entonces $2 k\left(1+x_{H 1}\right)=\left(1-c\left(x_{H 1}\right)\right)+1+c\left(x_{H 1}\right)=2$, lo que implica que $k_{H 1}=\frac{1}{1+x_{H 1}}$.

Sin embargo, si solo las condiciones $\mathrm{C} 1$ y $\mathrm{C} 3$ se satisfacen en igualdad, para cada valor de $c_{r}$ existe una única solución $x$ y $k$. Luego de un poco de algebra, $x\left(c_{r}\right)$ está definido por $-c^{\prime}(x)=\frac{1+c(x)}{2-x}+c_{r} \frac{2}{2-x}$ y el valor en $k$ es $\underline{k}_{H 2}=\frac{1+c(x)-x c^{\prime}(x)}{2}$.

Para cada $c_{r}$ el máximo valor de $k$ para que la empresa elija una estrategia de precio alto es

$$
\underline{k}_{H}\left(c_{r}\right)=\min \left\{-c^{\prime}\left(x_{H 1}\right)-c_{r}, \frac{1+c\left(x\left(c_{r}\right)\right)-x\left(c_{r}\right) c^{\prime}\left(x\left(c_{r}\right)\right)}{2}\right\}
$$

Dado $c_{r}$, la estrategia de precio alto puede ser óptima para todo $k \leq \underline{k}_{H}\left(c_{r}\right)$. Cuando $k$ aumenta la demand se desplaza hacia abajo. La empresa reduce el precio e incrementa la calidad. Para valores de $k$ mayores, los consumidores son más sensibles a la calidad y la empresa reduce $x$. Esta reducción en $x$ compensa parcialmente la caída en la demanda. Tanto el margen por unidad como las ventas caen y los beneficios de la empresa se reducen. Estos efectos definen una relación decreciente entre beneficios y el costo de reclamar de los consumidores representado por $\Pi_{H}$ en la figura 3.

\subsubsection{Estrategia de precio bajo}

Hay 3 condiciones necesarias para que la empresa elija una estrategia de precio bajo: C4- $-c^{\prime}(x)=$ $2 c_{r} \frac{(1-x)(1-k)}{(1-x)-c(x)}+\frac{1}{2}+\frac{c(x)}{2(1-x)}$ para el $x$ óptimo; C5- $\frac{(1-x-c(x))^{2}}{4(1-x)} \geq x c_{r}[1-k]$ para beneficios no negativos; y C6- $k \geq k_{L} \equiv \frac{1-x+c(x)}{2(1-x)}$ para satisfacer las restricciones. La condición C4 implica que la solución $x_{L}$ satisface $\frac{\partial x_{L}}{\partial k}>0$ y $\frac{\partial x_{L}}{\partial c_{r}}<0$ monotonicamente. Si la empresa sigue una estrategia de precio bajo los 


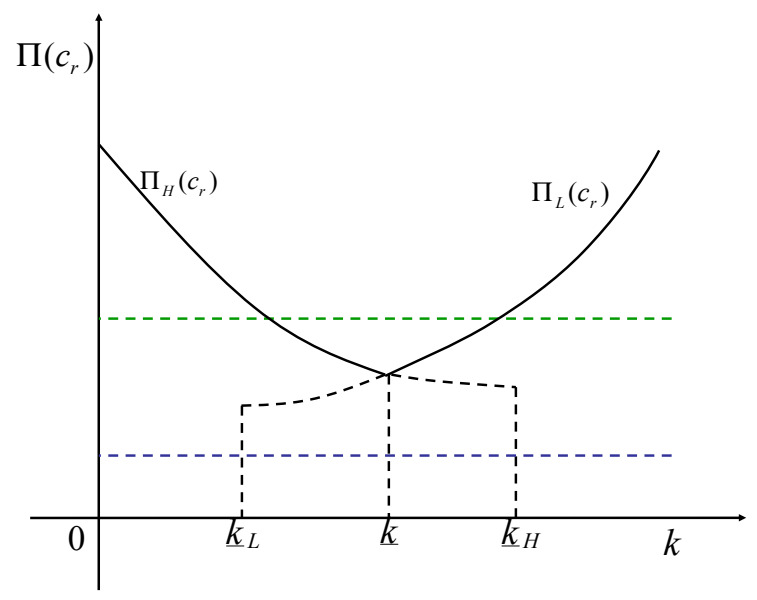

Figure 3: Beneficio máximo como función del costo de reclamar de los consumidores para estrategias de precio alto $(H)$ y bajo $(L)$

beneficios son

$$
\Pi=\frac{1}{1-x_{L}}\left(\frac{1-x_{L}-c\left(x_{L}\right)}{2}\right)^{2}-x_{L} c_{r}[1-k] \quad \text { si } k \geq \frac{1-x_{L}+c\left(x_{L}\right)}{2(1-x L)}
$$

Por el teorema de la envolvente $\frac{\partial \Pi}{\partial k}>0$ y $\frac{\partial \Pi}{\partial c_{r}}<0$ monotonicamente. Dado $c_{r}$ existe una cota inferior $\underline{k}_{L}\left(c_{r}\right)$ tal que para todo $k \geq \underline{k}_{L}\left(c_{r}\right)$ la función de beneficios satisface 8. También existe una costa superior $c_{r}$ para que la empresa tenga beneficios no negativos. Para encontrar la cota inferior $\underline{k}_{L}\left(c_{r}\right)$ analizamos los diferentes escenarios.

Si las condiciones C4 y C5 se satisfacen en igualdad, existe una relación entre $x, c_{r}$ and $k$ : dado $c_{r}$, existe $x_{L}$ y $k$ que satisfacen C4 y C5. De $x^{*} \equiv \arg \max \frac{1}{1-x}\left(\frac{1-x-c(x)}{2}\right)^{2}$ encontramos el valor de $x$ cuando $k$ es lo suficientemente tal que ningún comprador reclama. Si para $k$ alto la empresa tiene beneficios positivos reducimos el valor de $k$ hasta que la condición C5 se satisface. Si existe, $k_{L 1}\left(c_{r}\right)$ se encuentra en $(0,1)$ y está definido por $-c^{\prime}(x)=\frac{1}{2 x}\left[1-c(x) \frac{1-2 x}{1-x}\right]$. Si solo las condiciones $\mathrm{C} 4$ y C6 se satisfacen en igualdad tenemos la siguiente condición

$$
-c^{\prime}(x)=c_{r}+\frac{1}{2}+\frac{c(x)}{2(1-x)}
$$

De nuevo, el mínimo valor de $k$ que puede satisfacer ambas condiciones es $k_{L}=c\left(x_{L}\right)+x_{L} c_{r}$. Si $p=$ $k_{L}>c\left(x_{L}\right)+x_{L} c_{r}$, existe otro $\hat{k}$ con $\hat{x}$ and $\hat{p}$ óptimos tal que tenemos un equilibrio con $\hat{k}>\hat{p}$ y beneficio positivo.

El valor de $\underline{k}_{L}\left(c_{r}\right)$ está definido por

$$
\underline{k}_{L}\left(c_{r}\right)=\max \left\{k\left(c_{r}\right), c\left(x_{L}\right)+x_{L} c_{r}\right\}
$$

Cuando $k$ aumenta, el número de reclamantes entre compradores cae. La firma reduce tanto el precio 
como la calidad. Menos reclamos incentivan a reducir la calidad que, a su vez, desplaza la demanda hacia abjo. Una reducción en el precio compensa la reducción en las ventas. Finalmente, el margen es mayor y las ventas no caen significativamente. Estos efectos definen una relación positiva entre los beneficios y el costo de reclamar de los consumidores, representada por $\Pi_{L}$ en la figura 3.

\subsubsection{Cota superior en $c_{r 1}$ y valor crítico $\underline{k}$}

Probamos que la firma puede elegir una de dos tipos de estrategias, estrategia de precio alto o precio bajo. El siguiente lema establece la existencia del equilibrio no trivial.

Lemma 2. Dado A0- Al, existe $c_{r 1}$ tal que: si $c_{r} \leq c_{r 1}$ la solución al problema en la ecuación (4) existe y está caracterizada por una solución interior. De hecho, para todo $c_{r} \leq c_{r 1}$ existe un relacion $\underline{k}\left(c_{r}\right)$ tal que la estrategia óptima de la empresa se caracteriza por precio alto, $p>k(1-x)$, si $k<\underline{k}\left(c_{r}\right)$ y precio bajo, $p \leq k(1-x)$, en caso contrario.

El lema 2 dice que para valores muy altos de $c_{r}$ el problema no es interesante. Entonces podemos definir el conjunto $\Theta=[0,1] \times\left[0, c_{r 1}\right]$ como el conjunto de todas las combinaciones de $k$ y $c_{r}$ tal que existen equilibrios no triviales. Para este subconjunto de valores $\left(k, c_{r}\right)$ el lema confirma que la estrategia de equilibrio de la empresa depende de $\underline{k}\left(c_{r}\right)$. Existe un orden in $k$ de los equilibrios y existe un valor crítico $\underline{k}\left(c_{r}\right)$. Para $k \leq \underline{k}\left(c_{r}\right)$ la empresa elige una estrategia de precio alto y para $k>\underline{k}\left(c_{r}\right)$ la empresa elige una estrategia de precio bajo.

\section{Equilibro}

Nos concentramos en el concepto de Equilibrio Subjuego Perfecto de Nash. Cada agente elige su mejor respuesta en cada conjunto informativo.

Por el Lema 2, nos restringimos al conjunto $\Theta=[0,1] \times\left[0, c_{r 1}\right]$. Sin embargo, para valores de $c_{r}$ mayores que $c_{r 1}$ un equilibrio existe si $k$ es lo suficientemente grande. El siguiente lema establece este resultado.

Lemma 3. Dado AO y Al, para todo $c_{r}$ existe $k_{2}\left(c_{r}\right)$ tal que existe un equilibrio no trivial para todo $k \geq k_{2}$.

El Lema 3 establece que si $c_{r}$ es suficientemente grande existe un equilibrio solo si una cantidad suficientemente grande de consumidores nunca reclama compensación. En el límite, cuando $c_{r} \rightarrow+\infty$ existe un valor de $k_{2}$ tal que ningún consumidor reclamar y la empresa obtiene beneficios no negativos.

El Lema 2 nos permite caracterizar la estrategia óptima de la empresa dados $k$ y $c_{r}$. Para cada $c_{r}$, los beneficios son crecientes en $k$ para una estrategia de precio bajos y son decrecientes para una estrategia de precio alto. Una reducción marginal en el costo de reclamar reduce los beneficios si $k>\underline{k}\left(c_{r}\right)$ y los incremente si $k \leq \underline{k}\left(c_{r}\right)$. Para cualquier $k$, los beneficios se reducen monotonicamente con $c_{r}$. El siguiente lema garantiza que existe un valor crítico en $c_{r}$ por encima del cual la firma obtiene su máximo nivel de beneficios cuando $k=1$, y por debajo del cual la firma obtiene su máximo nivel de beneficios cuando $k=0$. 
Lemma 4. Dado AO y Al, existe $c_{r 2}$ tal que $0=\arg \max _{k} \Pi\left(p\left(k, c_{r}\right), x\left(k, c_{r}\right), k, c_{r}\right)$ si $c_{r}<c_{r 2}$ y $1=$ $\arg \max _{k} \Pi\left(p\left(k, c_{r}\right), x\left(k, c_{r}\right), k, c_{r}\right)$ si $c_{r 2}<c_{r}$. Esto significa que la firma obitene los máximos beneficios siguiendo un estrategia de precio alto cuando todos los consumidores reclaman con costo nulo si $c_{r} \leq c_{r 2}$ y sigiendo una estrategia de precio bajo sin reclamos si $c_{r}>c_{r 2}$.

La proposición 1 resume los resultado de los Lemas 1-4.

Proposition 1. Dado A0-A1, existe $c_{r 1}$ y una relación $\underline{k}\left(c_{r}\right)$ tal que para todo $\left(k, c_{r}\right) \in \Theta \equiv[0,1] \times\left[0, c_{r 1}\right]$ existe un equilibrio no trivial. No existe equilibrio tal que $p=0, p=1$ o $p=k(1-x)$ y existe $k\left(c_{r}\right)$ que divide al conjunto $\Theta$ en dos: para todo $\left(k, c_{r}\right) \in \Theta \equiv[0,1] \times\left[0, c_{r 1}\right]$ el equilibrio está caracterizado por precio bajo si $k \geq \underline{k}\left(c_{r}\right)$ y por precio bajo si $k \leq \underline{k}\left(c_{r}\right)$. La función beneficio indirecta es decreciente en $c_{r}, y$ tiene forma de $U$ en $k$ con el mínimo en $\underline{k}\left(c_{r}\right)$, y el máximo en $k=0$ sic $c_{r} \leq c_{r 2}$ y en $k=1$ si $c_{r} \geq c_{r 2}$.

Ya hemos definido y caracterizado el equilibrio. Definamos las implicancias de reducir el costo de reclamar de los consumidores en las variables principales.

Proposition 2. Dado A0-A1, existe $\Theta_{\Pi} \subset \Theta$ y $\Theta_{x} \subset \Theta$ tal que:

2.a- $\operatorname{Si}\left(k, c_{r}\right) \in \Theta_{\Pi}$ existe una reducción mínima de $k, \Delta \underline{k}$, tal que $\Pi(k-\Delta k)>\Pi(k)$ si $\Delta k \geq \Delta \underline{k}$, i.e. la empresa incrementa los beneficios cuando se reduce el costo de reclamar en al menos $\Delta \underline{k}$.

2.b- $\operatorname{Si}\left(k, c_{r}\right) \in \Theta_{x}$ existe una reducción mínima de $k, \Delta \underline{k}$, tal que $x(k-\Delta k)>x(k)$ si $\Delta k \geq \Delta \underline{k}$, i.e. la calidad del producto se reduce $(\Delta x>0)$ cuando se reduce el costo de reclamar de los consumidores en al menos $\Delta \underline{k}$.

Proof. Para la prueba se garantiza que existen $c_{r 2}$ y $c_{r 3}$ tal que $[0,1] \times\left[0, c_{r 2}\right] \subset \Theta_{\Pi}$ y que $[0,1] \times\left[0, c_{r 3}\right] \subset$ $\Theta_{x}$. Sin embargo, los conjuntos $\Theta_{\Pi}$ y $\Theta_{x}$ son mayores.

Para describir estos resultados analicemos primera el efecto de una reducción marginal en $k$. Bajo una estrategia de precio alto, una reducción marginal en $k$ genera una reducción en los costos de transacción. La empresa incrementa el precio y reduce la calidad. Tanto la demand como los beneficios aumentan. Bajo una estrategia de precio bajo, una reducción marginal en $k$ genera un incremento en los reclamos y en los costos de la firma. La empresa aumenta la calidad y el precio. El margen unitario y los beneficos caen. Ambos resultados son estándares en la literatura: en el primer caso la reducción del costo de transacción, en el segundo un incremento en los costos de producción.

Sin embargo, para una reducción discreta en $k$ tenemos un nuevo resultado. Para una estrategia de precio alto no hay nada nuevo. Para una estrategia de precio bajo, una reducción discreta en $k$ la firma genera un cambio de estrategia, cambiándose a una estrategia de precio alto. Un incremento sustancial en el precio genera una reducción en la demanda, un cambio en la calidad y un cambio en los beneficios. Cuando la reducción en $k$ es suficientemente grande tanto la calidad como los beneficios de la empresa caen. Esto se debe a que los consumidores encontraron un mecanismo barato que garantiza un buen producto la calidad pierde su valor: porque el producto no está fallado o porque hicieron un reclamo a bajo costo.

La proposición 2 describe claramente el resultado: una reducción suficientemente grande en el costo de reclamar genera una reducción en la calidad del producto y un incremento en los beneficios. La implicancia es clara: cualquier política que ayude a los consumidores a reclamar persigue que los productos 
sean de mejor calidad, pero el resultado puede ser el opuesto. Concomitantemente, los reclamos no son una buena señal de calidad.

Para ilustrar que nuestra proposición se mantiene bajo condiciones suficientemente generales observe las figuras 4 and 5. La línea roja separa el conjunto en dos, los valores para los cuales la empresa elige estrategia de precio bajo o estrategia de precio alto. Si la firma elige una estrategia de precio alto, cualquier reducción en el costo de reclamar genera un aumento en los beneficios y una reducción en la calidad (i.e., $\Delta x>0$ ). En la figura 4.a, la relación $\hat{k}_{\Pi}\left(c_{r}\right)$ define el valor máximo de $k$ tal que los beneficios puede aumentar en una estática comparativa. ${ }^{12}$ En la figura 4.b, la relación $\hat{k}_{x}\left(c_{r}\right)$ define el valor máximo de $k$ tal que la calidad del producto puede caer en una estática comparativa. ${ }^{13}$

Superponiendo las figuras 4.a y 4.b obtenemos la Figura 5 donde podemos definir los conjuntos $\Theta_{\Pi}$ y $\Theta_{x}: \Theta_{\Pi}=A \bigcup B \bigcup D$ y $\Theta_{x}=A \bigcup D$. Para cualquier dupla $\left(k, c_{r}\right)$ que pertenece a $A$, es necesario una reducción en $k$ de por lo menos $\Delta \underline{k}_{x}$ para que $\Delta \Pi>0$ y $\Delta x>0$. Si $\left(k, c_{r}\right)$ pertenece a $D$, sin embargo, cualquier reducción en $k$ genera $\Delta \Pi>0$ y $\Delta x>0$. Finalmente, si $\left(k, c_{r}\right)$ pertence a $C=\Theta / \Theta_{\Pi}$, cualquier reducción en $k$ genera $\Delta \Pi<0$ y $\Delta x<0$.

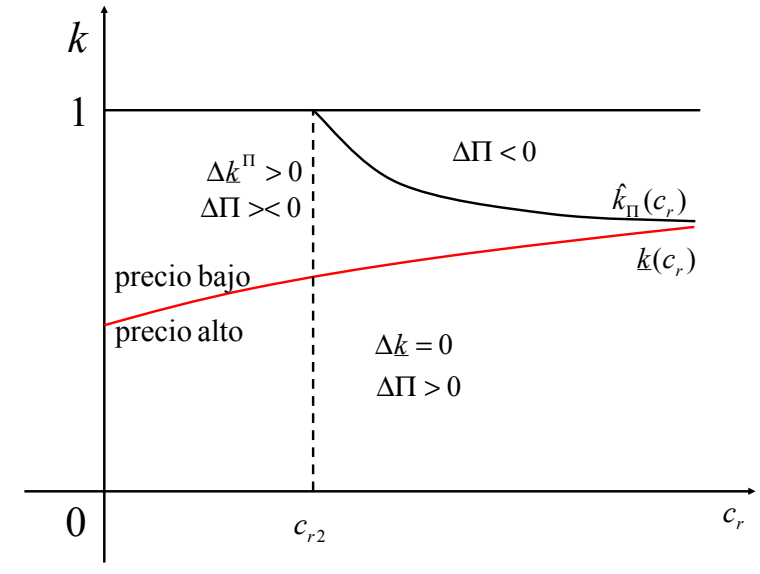

(a) Profit

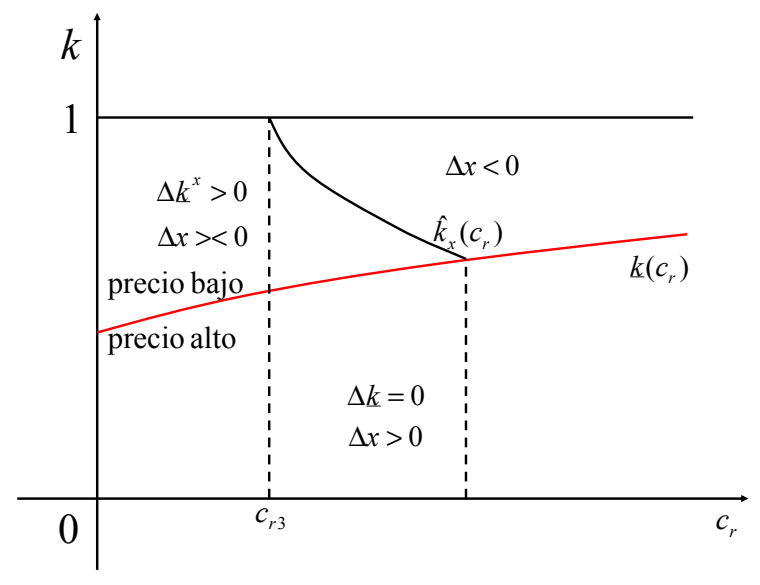

(b) Reliability

Figure 4: Parameters that can lead to an increase or decrease in profit (a) and reliability (b).

\footnotetext{
${ }^{12}$ En la siguiente relacion entre $k$ y $c_{r}$ tenemos que $x_{H}$ está definido por $-c\left(x_{H}\right)=c_{r}$ y que $x_{L}$ está definido por $-c^{\prime}\left(x_{L}\right)=$ $2 c_{r} \frac{\left(1-x_{L}\right)(1-\hat{k})}{\left(1-x_{L}\right)-c\left(x_{L}\right)}+\frac{1}{2}+\frac{c\left(x_{L}\right)}{2\left(1-x_{L}\right)}$.

$$
\hat{k}_{\Pi}\left(c_{r}\right)=\min \left\{\frac{1}{c_{r} x_{L}}\left[\frac{\left(1-c\left(x_{H}\right)-x_{H} c_{r}\right)^{2}}{4}-\frac{\left(1-x_{L}-c\left(x_{L}\right)\right)^{2}}{4\left(1-x_{L}\right)}\right]+1,1\right\}
$$

${ }^{13}$ Definamos el máximo $k$ tal que la calidad puede caer en una estática comparada$$
\hat{k}_{x}\left(c_{r}\right)=\min \left\{\frac{(1-x)^{2}-c(x)^{2}}{4 c_{r}(1-x)}+\frac{1}{2}+\frac{c(x)}{2(1-x)}, 1\right\}
$$

Donde $x$ está definido por $-c^{\prime}(x)=c_{r}$.
} 


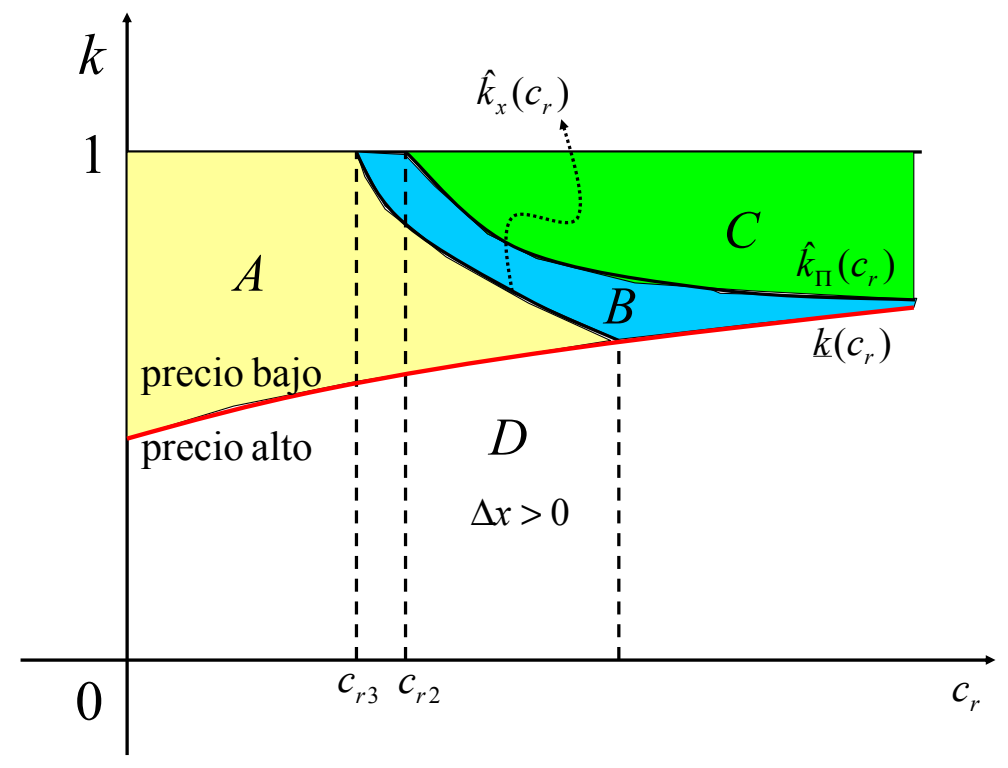

Figure 5: Set of parameters for joint variation in profits and reliability

\section{Ejemplo numérico}

Supongamos que la función de producción unitaria es $c(x)=\frac{\beta}{x},{ }^{14} \operatorname{con} \beta=0.01$. Es fácil verificar que esta función satisface los supuestos A0-A1. En las figuras 6 y 7 graficamos la tasa de fallos (calidad), el precio y los beneficios de la firma como una función del costo de reclamar de los consumidores, para diferentes valores de $c_{r}(\in\{0.2,0.5,0.8\})$. Primero note que el precio óptimo es decreciente en $k$ y tiene un salto que refleja el cambio en la estrategia de la empresa. Existe el valor crítico $\underline{k}$ por debajo del cuál la empresa elige una estrategia de precio alto. Tanto los beneficios de la empresa como la tasa de fallos tienen una forma en $\mathrm{U}$. El valor mínimo de los beneficios y la tasa de fallos coincide con $\underline{k}$.

Los valores críticos son $c_{r 1}=24.3, c_{r 2}=0.5593$ y $c_{r 3}=0.5426 .{ }^{15}$ Por ejemplo, una reducción en $k$ de 0.65 a 0.30 tiene un efecto negativo en los beneficios si $c_{r}=0.8$, un efecto positivo en los beneficios si $c_{r}=0.2$, y un efecto nulo si $c_{r}=0.5$. Por otro lado, un cambio en $k$ de 0.65 a 0.12 genera un reducción en la tasa óptima de fallos si $c_{r}=0.8$, un incremento en la tasa óptima de fallos si $c_{r}=0.2$, y no tiene efecto si $c_{r}=0.5$.

Resumiendo, la introducción de una asociación de consumidores, instituciones legales (pequeñas cortes y juicios en grupos), y cualquier mecanismo que ayude a los consumidores a realizar sus reclamos puede generar una reacción a favor o en contra por parte de las empresas dependiendo de la reducción en el costo de reclamar y en los costos que enfrenta la firma por esos reclamos. Una reducción en los costos de los reclamos genera una reducción en la calidad si los costos de los reclamos para la firma son suficientemente bajos.

\footnotetext{
${ }^{14}$ Esta función $c(x)=\frac{\beta}{x}$ satisface las especificaciones técnicas según Huang, Liu, and Murthy (2007).

${ }^{15}$ Para el caso donde los consumidores reciben un producto nuevo cuando reclaman los valores criticos son $c_{r 1}=23.3$, $c_{r 2}=0.4098$ y $c_{r 3}=0.3953$. En este caso los costos esperados por reemplazos enfrentados por la empresa son $x \frac{c(x)+c_{r}}{1-x} \frac{\hat{Q}(p, x)}{Q(p, x)}$.
} 


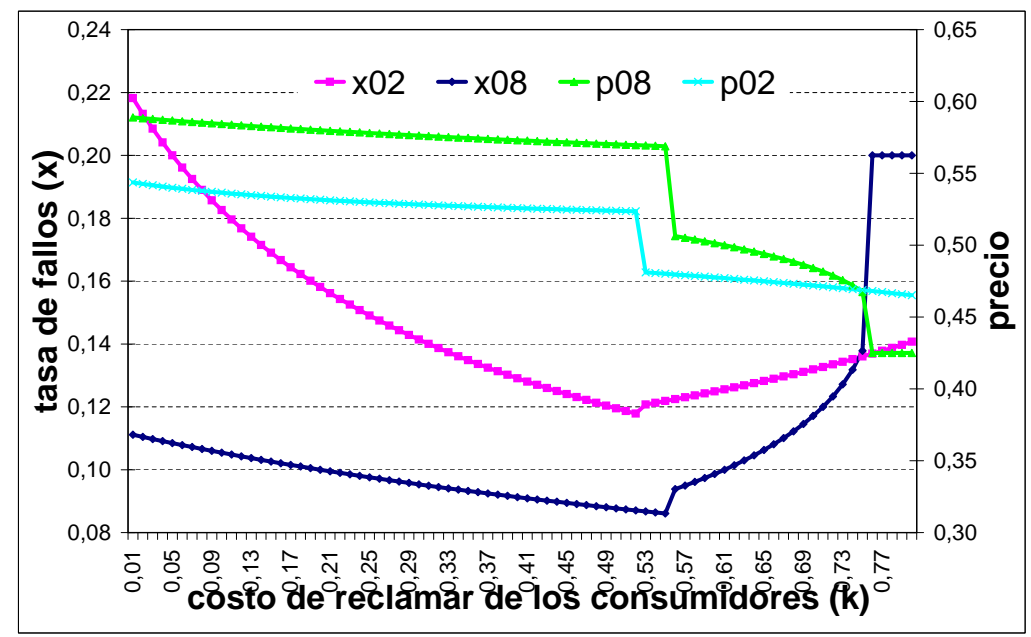

Figure 6: Precio y tasa de fallos de equilibrio como función del costo de los consumidores para reclar.

\section{El impacto de la Asociaciones de Consumidores}

Como mencionamos en la introducción, las asociaciones de consumidores (AC) han estado trabajando por el bien de los consumidores en diferentes dimensiones. Una de ellas es reducir el costo de reclamar de los consumidores para que estos ejerzan sus derechos como tales. In nuestro modelo estas AC afectan a los consumidores de dos maneras diferentes: i) moviendo la distribución de costos de reclamos hacia abajo, y ii) proveyendo una ayuda "legal" cuando los problemas aparecen (que es equivalente a proveer un menor costo de reclamar). In este trabajo, estos dos efectos no se pueden diferenciar.

En este modelo, todos los consumidores tienen el mismo costo de reclamar representado por $k$ (la distribución de costos de reclamar es degenerada en un punto). El costo de reclamar $k$ se reduce como consecuencia de la intervención de las asociaciones de consumidores, i.e. $k^{\prime}<k$. Nuestros resultados son robustos a una estructura más general donde existe heterogeneidad en el costo de reclamar entre los consumidores.

Por otro lado, nosotros esperamos que la intervención de las asociaciones de consumidores reduzca el costo de reclamar en una cantidad pequeña, i.e. marginal, o en una cantidad grande, i.e. discreta. Si el caso base es uno donde una proporción de compradores nunca reclama cuando recibe productos fallados, el efecto de las AC es claramente mejorar la calidad en el primer caso (reducción marginal de $k$ ) e indefinido en el segundo caso (reducción discreta de $k$ ). Nuestra principal contribución es que el cambio discreto en el costo de reclamar de los consumidores puede generar una reducción en la calidad del producto.

En el mercado de autos de U.S.A, The National Highway Traffic Safety Administration (NHTSA) es una agencia gubernamental que tiene la autoridad de reglamentar los estándares de seguridad de los vehículos y de requerir a las empresas a realizar recalls de los vehículos que tengan defectos relacionados con seguridad o no cumplan la normativa federal de seguridad. Véase Rupp and Taylor (2002) para una explicación detallada del proceso de recalls. Los consumidores utilizan el servicio de la NHTSA como un mecanismo adicional para expresar sus reclamos. Si la compañía no atiende sus requerimientos, los 


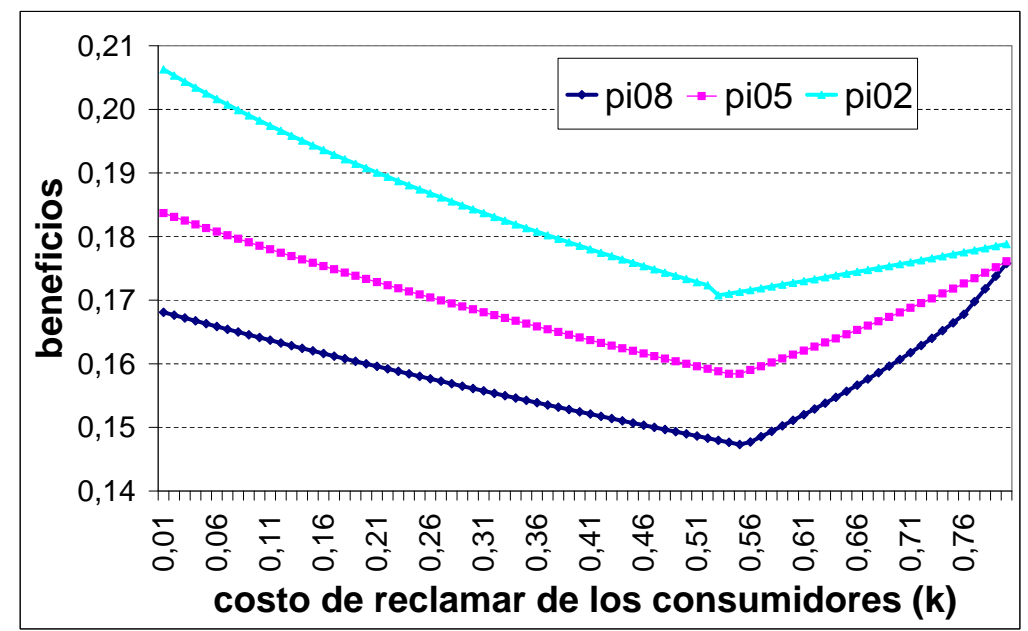

Figure 7: Beneficios de equilibrio como función del costo de los consumidores para reclar

consumidores eligen entre demandar privadamente a la empresa, contactar una agencia de consumidores (e.g. Consumer Union in U.S.A.), hacer una queja a través de la NHTSA, una combinación de las anteriores, o no hacer nada.

Como mostramos en la introducción, se observa que el número de recalls se relaciona positivamente con el número de quejas desde que los consumidores expresen sus reclamos a través de la NHTSA. Este indicio es consistente con los siguiente efectos: las compañias automotrices bajaron la calidad de los producots, la NHTSA endureció los controles y/o normativas de seguridad, los consumidores ayudan a revelar más información, o, más probablemente, una combinación de todas las anteriores.

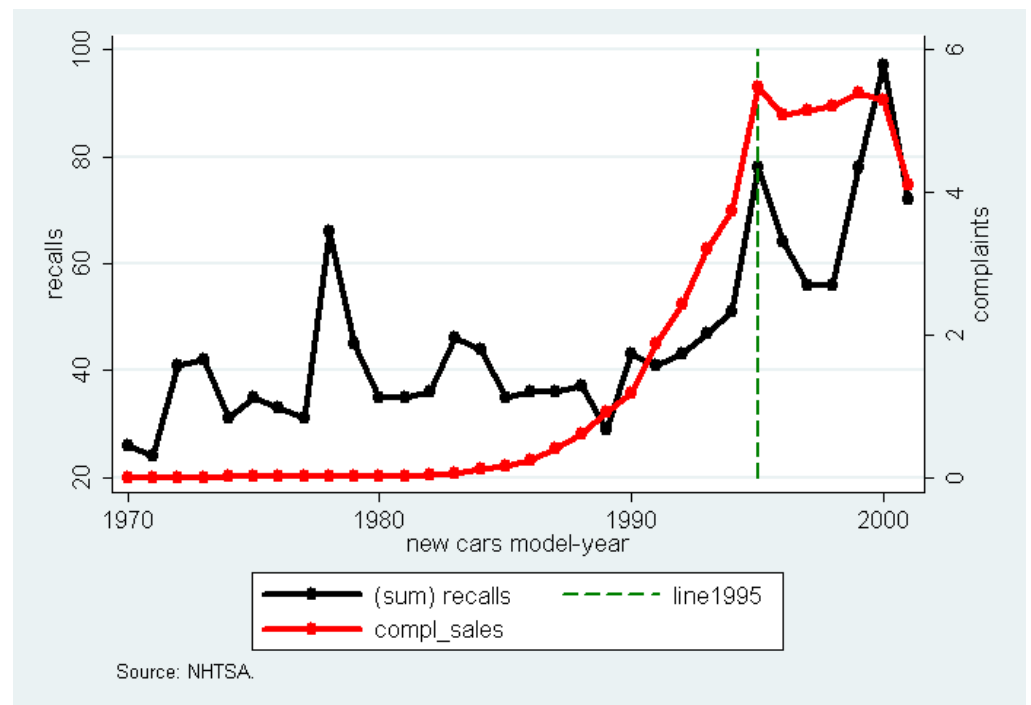

Figure 8: Número de Recalls y Quejas en el mercado automotriz de U.S.A. por modelo de vehículo

Supongamos que la NHTSA ha endurecido sus controles y los ha aplicado de form retrospectiva 
Table 1: Número de recalls por modelo-año y la distribución en el tiempo.

\begin{tabular}{c|ccccc} 
& \multicolumn{5}{|c}{ Tiempo transcurrido desde lanzamiento } \\
vehículos producidos entre & recalls & mismo año & $1-5$ años & $6-10$ años & resto \\
\hline $1977-1982$ & $\mathbf{2 4 8}$ & $73.39 \%$ & $22.58 \%$ & $\mathbf{3 . 6 3} \%$ & $0.40 \%$ \\
$1983-1988$ & $\mathbf{2 3 4}$ & $52.14 \%$ & $39.32 \%$ & $\mathbf{8 . 1 2} \%$ & $0.43 \%$ \\
$1989-1994$ & $\mathbf{2 5 2}$ & $44.05 \%$ & $40.87 \%$ & $\mathbf{1 2 . 7 0} \%$ & $2.38 \%$ \\
$1995-2000$ & $\mathbf{4 2 9}$ & $50.12 \%$ & $44.76 \%$ & $\mathbf{5 . 1 3} \%$ & -
\end{tabular}

en 1995, que hay mayor revelación de información pero que la calidad de los vehículos permaneció constante. Si este es el caso, debemos observar que el número de recalls para los autos producidos alrededor de 1995, tanto antes como después, es similar. Sin embargo, la serie de la Figura 8 muestra que el número de recalls es considerablemente mayor para los vehículos producidos luego de 1995. Este hecho es consistente con nuestra predicción que la reducción en el costos de reclamar motiva a las empresas a reducir la calidad de los productos manufacturados.

La Tabla 1 muestra el número de recalls por modelo agregando en períodos de 6 años y su distribución cronológica. Los autos producidos entre 1995 y 2000 sufrieron 429 recalls, y la mitad de ellos ocurrieron durante el primer año de lanzamiento. Note que luego de 1995 hay un incremento importante en el número de recalls, de 42 para los vehículos producidos en 1994 a más de 70 en 1995.

Anteriormente afirmamos que las quejas de los consumidores ayudan a revelar más información. Consistente con nuestro postulado, hay una una evidencia clara que los dispositivos on-line de la NHSTA ayudaron a revelar más información: en los periodos 1977-1982 y 1995-2000 entre el 4 y el 5\% de todos los recalls ocurrieron en el sexto año desde que los vehículos fueron lanzados al mercado o posteriormente (columna 5 y 6 en la Tabla 1). Esta proporción se eleva a $8.5 \%$ y $15 \%$ para los vehículos manufacturados entre 1983-1988 y entre 1989-1994, respectivamente. Nueva información permitió muchos recalls que no hubiesen sido realizados de otra forma. ${ }^{16}$ Este hecho sugiere que sin las quejas de los consumidores, la tendencia en los recalls hubiera sido decreciente.

Concluyendo, observamos que hay una evidencia clara del efecto de los dispositivos on-line de la NHTSA en las quejas y en los recalls. Este dispositivo que abarata realizar quejas tuvo su efecto incrementando el número de recalls. Nosotros postulamos que estos incrementos se deben a mayor revelación de información, a un control más fuerte de la NHTSA y a una reducción el a calidad de los vehículos. El incremento en el número de recall por modelo-año luego de 1995 concilia nuestro resultados.

\section{Conclusiones}

Hemos demostrado que el impacto de las asociaciones de consumidores de reducir el costo de reclamar de los consumidores puede ser tanto incrementar o reducir la calidad de los productos. Este resultado is obtenido asumiendo heterogeneidad en las valuaciones de los consumidores por los productos sin fallas. Este supuesto es suficiente para vincular el precio con la proporción de reclamantes y no reclamantes entre los compradores. Esta relación se transmite también al costo marginal agregado, generando una

\footnotetext{
${ }^{16} \mathrm{Si}$ consideramos solo los recalls dentro de los primeros 5 años desde que los vehículos fueron lanzados, el incremento en los recalls de 1989-1994 a 1995-2000 es de 214 a 409 recalls, casi un 100\% de incremento.
} 
relación entre el costo de reclamar de los consumidores, el precio y la calidad del producto.

La contribución principal de este trabajo son levantar un aviso de los efectos de reducir el costo de reclamar de los consumidores en al calidad del producto y proveer una relación entre el precio del producto y los reclamantes.

Hay dos extensiones relevantes. Por un lado, introducir una dinámica puede explotar los efectos de los costos de firma ligados a los reclamos y su relación con los costos de reclamar de los consumidores. Por otro lado, modelar el comportamiento estratégico de mercado entre diferentes empresas puede vislumbrar nuevos indicios sobre el efecto del costo de reclamar de los consumidores, la calidad del producto y la performance del mercado.

\section{Apéndice}

\subsection{Supuesto A1}

El supuesto A1 es fácil de entender pero es más fuerte de lo que realmente se necesita. Lo que se necesita es lo siguiente: recordemos que $x_{0}$ se obtiene de $-c^{\prime}\left(x_{0}\right)=\frac{1}{2}+\frac{c\left(x_{0}\right)}{2\left(1-x_{0}\right)}$ y maximiza $\Pi=\frac{(1-x-c(x))^{2}}{4(1-x)}$. Definamos el beneficios máximo como $\Pi_{0}$. Necesitamos que cuando $c_{r}=0$ ambos beneficios se igualen por lo menos en algún valor $k \in(0,1)$, i.e. $\Pi_{L} \equiv \frac{(1-x-c(x))^{2}}{4(1-x)}=\Pi_{H} \equiv \frac{(1-c(x)-x \hat{k})^{2}}{4}$.

Requerimos que exista $x$ tal que $1-c(x)-2 \sqrt{\Pi_{0}}>0$.

NOTA: el supuesto A1 no es necesario, con $1-x_{0}-c\left(x_{0}\right)>0$ es suficiente. Para que $\hat{k}$ exista es suficiente con probar que $\Pi_{L} \equiv \frac{(1-x-c(x))^{2}}{4(1-x)}=\Pi_{H} \equiv \frac{(1-c(x))^{2}}{4}$ y que $\Pi_{H}$ es creciente en $k$ y que $\Pi_{H}(k=$ 1) $<\Pi_{0}$.

\subsection{Prueba del Lema 1}

Proof. La prueba del Lema 1 consiste en mostrar que algunas condiciones necesarias para que $p=c$ sea un equilibrio generan una contradicción. Es trivial mostrar que en $p=0$ la firma obtiene beneficios negativos y que en $p=1$ la firma tiene beneficios nulos. Si $p=c$ es un equilibrio la función beneficios no debe incrementar ni por la izquierda ni por la derecha. La derivada primera respecto del precio evaluada en $p=c$ es

$$
\begin{array}{r}
\frac{\partial \Pi}{\partial p}=[1-k(1+x)]-\left(k-c(x)-x c_{r}\right) \leq 0 \quad \text { si } \quad k<p, \mathrm{y} \\
\frac{\partial \Pi}{\partial p}=\left[1-\frac{k}{1-x}\right]-\frac{(k-c(x))}{1-x} \geq 0 \quad \text { si } \quad k \geq p .
\end{array}
$$

pero para satisfacer estas condiciones simultáneamente encontramos la siguiente contradicción,

$$
0=[1-k]-[1-k] \leq k x+k-c(x)-x c_{r}-(x+k-c(x))<0 .
$$

La prueba es completa. 


\subsection{Prueba del Lema 2}

Proof. Dado $c_{r}=0, \underline{k}_{L}$ y $\underline{k}_{H}$ representan la restricción para $x$ en cada estrategia. La condición de primer respecto de $x$ para la estrategia de precio alto es $-c^{\prime}\left(x_{H}\right)=\frac{1+c\left(x_{H}\right)}{2-x_{H}}$ y para la estrategia de precio bajo es $-c^{\prime}\left(x_{L}\right)=\frac{c\left(x_{L}\right)}{\left(1-x_{L}\right)}$.

FALTA PROBAR QUE $x_{L}>x_{H}$. La desigualdad $x_{L}\left(c_{r}=0\right)>x_{H}\left(c_{r}=0\right)\left(1-x_{L}<1-x_{H}\right)$ se mantiene ya que $\frac{c(x)}{1-x}<\frac{2 c(x)}{(1-x)(2-x)}+\frac{1+c(x)}{2-x}$. Hemos probado que al menos para $c_{r}=0$ existe un equilibrio donde $\underline{k}_{L}<\underline{k}_{H}$. Reemplazando las expresiones necesitamos que se cumpla la siguiente condición

$$
\begin{array}{r}
\frac{c\left(x_{L}\right)}{\left(1-x_{L}\right)}<\frac{1+c\left(x_{H}\right)}{2-x_{H}} \\
-\left(x_{L}-x_{H}\right)-x_{L}\left(1+x_{H}-c\left(x_{L}\right) \frac{x_{H}}{x_{L}}\right) \leq 2\left[c\left(x_{H}\right)-c\left(x_{L}\right)\right]
\end{array}
$$

Para que se mantenga una estrategia de precio bajo necesitamos que $k_{L}<1-x_{L}$. Como $x_{L}=$ $\arg \min \frac{c(x)}{(1-x)}$ la condición (15) se mantiene. Finalmente, note que la función de beneficios para cada estrategia separada es monotonica en $k$ y la función de beneficios es continua en $k$.

Esto implica que existe $c_{r 1}>0$ tal que para todo $c_{r}<c_{r 1}$ el equilibrio no trivial siempre existe. En la Figura 3.b graficamos la función de beneficios para cada estrategia. La empresa elige la estrategia que le proporciona mayores beneficios. Hemos mencionado las condiciones que garantizan $\underline{k}_{L}<\underline{k}_{H}$. Esta condición no solo garantiza que el equilibrio existe, sino que también existe $\underline{k}$ tal que elige una estrategia de bajo precio si $k>\underline{k}$ y elige una estrategia de precio alto si $k<\underline{k}$.

\subsection{Prueba del Lema 3.}

Proof. Cuando $k \sim 1$ los beneficios de la empresa son $\Pi=\left(\frac{1-x-c(x)}{2}\right)^{2} \frac{1}{1-x}$, los cuales son positivos por los supuestos A0-A1 y no dependen de $c_{r}$ ni $k$. Además, por teorema de la envolvente, tenemos que $\frac{d \Pi}{d c}>0$ y $\frac{d \Pi}{d c_{r}}<0$. Supongamos que $c_{r}$ es extremadamente grande, entonces para todo $k$ suficientemente grande tenemos un equilibrio por lo menos si $k \sim 1$. Por continuidad, cuando $c_{r}$ cae $\Pi$ aumenta $\mathrm{y}$ podemos reducir el mínimo $k$ necesario para que el equilibrio exista. Obviamente este lema no garantiza que el equilibrio pueda existir para todo $k \in[0,1]$ pero al menos para un subconjunto el equilibrio no trivial existe.

\subsection{Proof of Lemma 4}

Proof. Cuando $k \sim 1$ los beneficios de la empresa son $\Pi_{0}=\left(\frac{1-x-c(x)}{2}\right)^{2} \frac{1}{1-x}$. Este valor de los beneficios es independiente de $c_{r}$. Sin embargo, cuando $k=0$ la función de beneficios es $\Pi_{H}\left(c_{r}\right)=$ $\left(\frac{1-c(x)-x c_{r}-x k}{2}\right)^{2}$. Aplicando el teorema de la envolvente tenemos que $\frac{d \Pi_{H}}{d c_{r}}<0$. Gracias a los supuestos A0-A1 tenemos que $\Pi_{0}<P i_{H}\left(c_{r}=0\right)$. Por continuidad de la función de beneficios indirecta $\Pi_{H}$, existe $c_{r 2}$ tal que $\Pi_{0}=P i_{H}\left(c_{r 2}\right)$, y la prueba es completa.

$\Pi_{H}\left(c_{r}\right)=\left(\frac{1-c(x)-x c_{r}-x k}{2}\right)^{2}$. Applying envelope theorem we have that $\frac{d \Pi_{H}}{d c_{r}}<0$. By assumption A0A2 we get that $\Pi_{0}<P i_{H}\left(c_{r}=0\right)$. By continuity of indirect profit function $\Pi_{H}$ there exists $c_{r 2}$ such that 
$\Pi_{0}=P i_{H}\left(c_{r 2}\right)$, and the proof is complete.

Para calcular el valor de $c_{r 2}$ vea la siguiente fórmula.

$$
c_{r 2}=\frac{1}{x_{0}}\left[1-c\left(x_{0}\right)-\frac{1-x_{0}-c\left(x_{0}\right)}{\sqrt{1-x_{0}}}\right]
$$

\subsection{Prueba de la Proposición 2}

Probemos primer Proposición 2.a

Proof. De la prueba del Lema 4 (o función de beneficio indirecta bajo la estrategia de precio alto) tenemos que $\frac{d \Pi_{H}}{d c_{r}}<0$ y $\frac{d \Pi_{H}}{d c}<0$. Cuando la empresa sigue una estrategia de precio bajo, la función de beneficios indirecta satisface $\frac{d \Pi_{L}}{d c_{r}}<0$ and $\frac{d \Pi_{L}}{d c}>0$. Probamos que existe un valor crítico $\underline{k}$ por encima del cual la firma prefiere una estrategia de precio bajo y por debajo del cual la empresa elige una estrategia de precio alto. La función de beneficios es continua en $k$.

Dado $c_{r}<c_{r 2}$, la empresa maximiza beneficios cuando $k=0$. Entonces, para cada $k>\underline{k}$ existe $k^{\prime}<\underline{k}$ tal que $\Pi_{L}(k)=\Pi_{H}\left(k^{\prime}\right)$. Por continuidad de $\Pi_{H}$ el beneficio es estrictamente mayor para todo $k$ tal que $k<k^{\prime}$.

Dado $c_{r}>c_{r 2}$ la empresa maximiza beneficios cuando $k=1$. Entonces, para cada $k<\underline{k}$ existe $k^{\prime}>\underline{k}$ tal que $\Pi_{L}\left(k^{\prime}\right)=\Pi_{H}(k)$. Por continuidad de $\Pi_{L}$ el beneficio es estrictamente mayor para todo $k$ tal que $k>k^{\prime}$.

Probemos ahora Proposición 2.b

Proof. Cuando $k \sim 1$ el valor de $x_{L}$ está definido por $-c^{\prime}\left(x_{L}\right)=\frac{1}{2}+\frac{c\left(x_{L}\right)}{2\left(1-x_{L}\right)}$ el no depende de $c_{r}$. Sin embargo, para $k<1$ la estrategia de precio bajo depende de $k$ and $c_{r}, \frac{d x_{L}}{d k}>0 \mathrm{y} \frac{d x_{L}}{d c_{r}}<0$.

Cuando $k=0$ el valor de $x_{H}$ está definido por $-c^{\prime}\left(x_{H}\right)=c_{r}+k$. De esta ecuación tenemos que $\frac{d x_{H}}{d k}<0$ y $\frac{d x_{H}}{d c_{r}}<0$. Bajo los supuestos A0-A1 tenemos que $x_{L}>x_{H}\left(c_{r}=0, k=0\right)$. Por continuidad de $x_{H}\left(c_{r}, k\right)$, existe $c_{r 3}$ tal que $x_{L}=x_{H}\left(c_{r 3}, k=0\right)$.

El valor óptimo de $x$ es continuo en $k$ excepto en $\underline{k}$. Sin embargo, siguiendo la proposición podemos probar que para cualquier $k^{\prime} \in(0,1]$, existe $k^{\prime \prime}$ tal que $x\left(k^{\prime \prime}\right)>x\left(k^{\prime}\right)$ si $c_{r}<c_{r 3}$, y la prueba es completa.

El valor de $c_{r 3}$ se puede calcular de la siguiente ecuación,

$$
c_{r 3}(c(x)) \equiv \frac{1-x_{0}+c\left(x_{0}\right)}{2\left(1-x_{0}\right)}
$$

donde $x_{0}$ se obtiene de $-c^{\prime}\left(x_{0}\right)=\frac{1-x_{0}+c\left(x_{0}\right)}{2\left(1-x_{0}\right)}$. Note que $c_{r 3}$ es una cota inferior para que la calidad caiga cuando hay una reducción en $k$, i.e. a menor $k$ mayor el $c_{r}$ compatible con una caída en la calidad luego de una reducción en el costo de reclamar de los consumidores (para algunos $c_{r}>c_{r 3}$ también $\Delta x>0$ cuando $\Delta k<0$ ). Esto implica que existe una mayor probabilidad de que haya una reducción en la calidad cuando las asociaciones de consumidores intervienen en el mercado. 


\section{References}

BAR-IsAaC, H., G. CARUANA, AND V. CUÑAT (2009): “Information Gathering and Marketing," Journal of Economy and Marketing Strategy, forthcoming.

BEst, A., AND A. R. ANDREASEn (1977): "Consumer Response to Unsatisfactory purchases: a survey of perceiving defects, voicing complaints, and obtaining redress," Law and Society Review, 11, 701.

Chebat, J.-C., M. Davidow, And I. Codjovi (2005): "Silent Voices: Why some Dissatisfied Consumers Fail to Complain,” Journal of Service Research, 7, 328-333.

Daughety, A. F., And J. F. Reinganum (1995): "Product Safety: Liability, R \& D, and Signaling," American Economic Review, 85, 1187 - 1206.

_ (2005): “Secrecy and Safety," American Economic Review, 95, 1074 - 1091.

- (2008): "Products Liability, Signaling and Disclosure," Journal of Institutional and Theoretical Economics, 164, 106 - 126.

Greif, A., P. Milgrom, And B. R. Weingast (1994): “Coordination, Commitment, and Enforcement: The Case of the Merchant Guild," Journal of Political Economy, 102, 433-776.

Hensler, D. R. (2001): "Revisiting the Monster: New Myths and Realities of Class Action and other Large Scale Litigation,” Duke Journal of Comparative and International Law, 11, 179.

HuAnG, H. Z., Z. J. LiU, AND D. N. P. Murthy (2007): "Optimal reliability, warranty, and price for new products," IIE Transactions, 39, 819 - 827.

HUPPERTZ, J. W. (2007): “Firms' complaint handling policies and consumer complaint voicing," Journal of Consumer Marketing, 24, 428 - 437.

Inderst, R., And M. OtTAViAni (2009): "Sales Talk, Cancellation Terms, and the Role of Consumer Protection," Working paper.

Klein, B., And K. B. LefFler (1981): "The Role of Market Forces in Assuring Contractual Performance," Journal of Political Economy, 89, 615-641.

Klement, A., and Z. Neeman (2004): "Incentive Structure for Class Action Lawyers," Journal of Law and Economics and Organization, 20.

MuRThy, D., AND I. DJAMALudin (2002): "New product warranty: A literature review," International Journal of Production Economics, 79, 231-260.

OI, W. Y. (1973): "The Economics of Product Safety," The Bell Journal of Economics and Management Science, 4, 3 - 28.

Owens, D. L., AND D. R. HAusKnecht (1999): "The effect of simplifying the complaint process: a field experiment with the Better Business Bureau," Journal of Consumer Satisfaction, Dissatisfaction, and Complaining Behavior, 12, 35-43. 
Rupp, N. G., AND C. R. TAYLOR (2002): "Who Initiates Recalls and Who Cares? Evidence from the Automobile Industry," Journal of Industrial Economics, 50, 123 - 149.

XiNYU, H. (Forthcoming JLEO): "Proudct Recall and Liability," Journal of Law, Economics and Organization. 